\title{
Modified Particle Swarm Optimisation for Economic Load Dispatch Problem
}

\author{
Ashita Dutta, Ann Susan Luke, Samya Kumar Gupta, P. Tejasree, Shivam Shukla, \\ Vishwajeet Kumar, P. Suresh
}

\begin{abstract}
For every Power System the concept of Economic Load Dispatch has and continues to be an area of immense importance and concern. With the steady increase in demand over the years it has become all the more mandatory to reduce the cost of power generation and at the same time meet all the power requirements of the concerned area. There are a number of techniques to solve the ever existing problem of Economic Load Dispatch such as Bat Algorithm, Firefly Method, NewtonRaphson Method, Lambda Iterative Method and so on and so forth. Each of these heuristic approaches require the cost curve to be piece wise linear. In this paper, Modified Particle Swarm Optimization has been proposed to find the most economical and ideal solution to the load dispatch problem. This is done by focussing on the population size of the solution space more than on other cost parameters. The simulation for the system is performed on MATLAB software.
\end{abstract}

Keywords--- PSO, ELD, Ramp Limit Constraints, Inertia Weight Factor.

\section{INTRODUCTION}

Economic Load Dispatch, a major concern in Modern Power Systems, is the process of designating particular levels of generation to each of the available generating units in such a way that the ideal solution is achieved as well it is cost effective. This is not a new concept; it has been an area of importance for a long time but with the technological advancements today it is of much higher concern than ever. Economic Dispatch develops a model for the entire Power System with respect to a set of predefined constraints. Based on this model it dispatches the required generation resources to full fill the demand. Cost curves and cost functions are quadratic in nature.

All the operation is done in real-time in accordance with the frequently changing power demand. While deriving the perfect solution for each instantaneous power demand $\left(\mathrm{P}_{\mathrm{d}}\right)$ it is imperative to maintain the overall system stability. Till

Revised Manuscript received July 10, 2019.

Ashita Dutta, Student, Department of Electrical and Electronics Kanchipuram Dist. T.N, India.

Ann Susan Luke, Student, Department of Electrical and Electronics Engineering, SRM Institute of Science and Technology, Kattankulathuur, Kanchipuram Dist. T.N, India.

Samya Kumar Gupta, Student, Department of Electrical and Electronics Engineering, SRM Institute of Science and Technology, Kattankulathuur, Kanchipuram Dist. T.N, India.

P. Tejasree, Student, Department of Electrical and Electronics Engineering, SRM Institute of Science and Technology, Kattankulathuur, Kanchipuram Dist. T.N, India.

Shivam Shukla, Student, Department of Electrical and Electronics Engineering, SRM Institute of Science and Technology, Kattankulathuur, Kanchipuram Dist. T.N, India.

Vishwajeet Kumar, Student, Department of Electrical and Electronics Engineering, SRM Institute of Science and Technology, Kattankulathuur, Kanchipuram Dist. T.N, India.

P. Suresh, Assistant Professor, Department of Electrical and Electronics Engineering, SRM Institute of Science and Technology, Kattankulathuur, Kanchipuram Dist. T.N, India. Engineering, SRM Institute of Science and Technology, Kattankulathuur,

date many techniques and algorithms have been designed to solve this optimization problem. Some of the oldest algorithms in use like the Lambda Iterative method, the gradient method, dynamic coding, the NR method, have a complex process flow. This thereby increases the time of computation. The linear programming approach in comparison to the above mentioned is faster but in this case the limits are only applicable to the positive variables. In recent times researchers all over the world have come up with more time effective and simpler methodologies such as the Tabu search technique, Genetic algorithm method(GA) and Artificial Neural Network based techniques. Bat and Novel Bat methods have also been developed and in use which are based on the typical behaviour of a bat and how it moves .

As the world is getting more and more concerned with the health of the environment around them, dispatching power is being made eco-friendlier and for this purpose algorithms are being developed. Particle Swarm Optimization is a process where every plausible solution to a given problem is taken to be a particle and each particle has its own position vector and velocity vector. The algorithm mainly focusses on finding the

best of all the available particles by putting up certain constraints. As compared to all the above mentioned methods available for economic load dispatch, PSO takes lesser time to deliver the result as the operation focusses on few important parameters rather than all. The Modified Particle Swarm Optimization presented in this paper further reduces the computation time of the algorithm as the main focus is on optimizing the population size with respect to the inertia weight constant and the damping factor.

Any modern power system invariably has more than one generator installed in it and every generator has its own particular set of operating parameters i.e. cost per hour characteristics as depicted in Figure 1. Thus each of these generators will be able to work within a pre-defined range $\left(\mathrm{P}_{\min }\right.$ and $\left.\mathrm{P}_{\max }\right)$. Based on this the generator scheduling is done.

\section{ECONOMIC LOAD DISPATCH}

Economic Load Dispatch (ELD) is lucidly defined as a technique that is applied to power systems by specifying different generation levels to various generating units in a manner where supply to the complete system load is ensured and that the process of generation and distribution is carried out in the most economically sustainable structure.

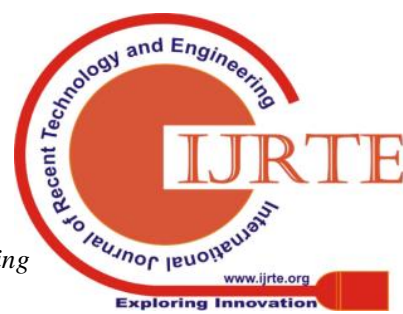


Lessening the expense is a crucial aspect for grid (interconnected) systems. The main purpose of this technique is to define production levels of individual plants so the price of generating and transmitting power is the least possible value for the prescribed load. In this manner, the total cost is reduced.

A few conventionally used methods or algorithms are Lagrange multiplier, Newton-Raphson and Lambda-iterative methods. These offer a tedious and time-consuming approach to solving standard ELD problems upon a variation in the load. Each computational loop calculates ELD of the system at each instant, thus resulting in a depreciation in the efficiency. The computational loop involves distributing the total power to be generated among several operating generation units. The selected cost criteria are minimized to the maximum possible extent by taking into account various operating parameters. Additionally, load and operational constraints are applied.

The cost is approximated by means of a few segments. For active power generation, the fuel cost can be estimated using a quadratic form as given in equation (1), where $a_{i}, b_{i}$ and $c_{i}$ are cost coefficients defined for the $i^{\text {th }}$ generating unit, $\mathrm{F}_{\mathrm{i}}$ is the total generational cost.

The cost coefficients are specified as fixed percentages of the incoming fuel costs. The sequential increase of power output of fossil plants can be attributed to the opening of valves leading to the steam turbine at the inlet.

During initial conditions, when the valves are just opened throttling losses are relatively high. The progressively decrease and are lowest upon complete opening of the valves.

ELD problems are generally non-linear programming optimization problems.

Under various system and operational constraints, optimum schedules of generating units are calculated so as to satisfy the load demand while observing minimum operational cost. The significance of ELD is the acquirement of maximum useful power whilst making use of minimum resources.

ELD problems of power systems are typically solved in a domain of unit commitment and real-time operation after giving relevance to the fact that every dispatch able active unit can be continuously regulated between the range of its minimum $\left(\mathrm{P}_{\min }\right)$ and its maximum $\left(\mathrm{P}_{\max }\right)$ generation limit

\section{POWER GENERATION COST OF THERMAL POWER PLANT}

In a power system the main factors which affect the generation cost per unit are the losses in the transmission line, efficiency of each and every generator and the fuel cost of every unit.

The cost function is such that it depends upon the individual cost of generation which is defined by some constraints under the specific limits and values.

The major hindrance is to at all times satisfy each of the given constraints. In doing so, some compromise has to be made.

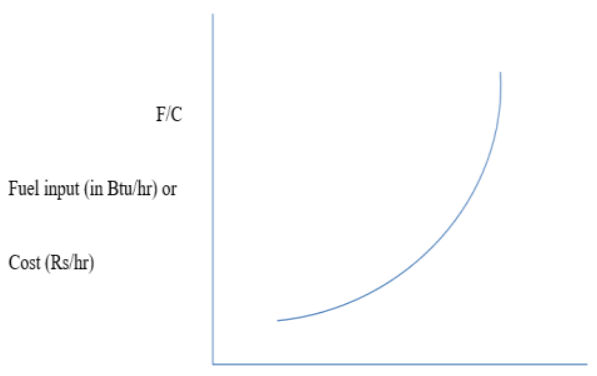

Fig. 1: Cost Curve of generator unit

In all practical problems the fuel cost of the ' $i^{\text {th }}$, generating unit is represented as shown in equation (1). This equation is used to determine the real cost of power generation.

$$
\mathrm{F}_{\mathrm{i}}\left(\mathrm{P}_{\mathrm{Gi}}\right)=\mathrm{a}_{\mathrm{i}} \mathrm{P}_{\mathrm{Gi}}{ }^{2}+\mathrm{b}_{\mathrm{i}} \mathrm{P}_{\mathrm{Gi}}+\mathrm{Ci}
$$

Where $\mathrm{F}_{\mathrm{i}}\left(\mathrm{P}_{\mathrm{i}}\right), \mathrm{P}_{\mathrm{i}},\left(\mathrm{a}_{\mathrm{i}}, \mathrm{b}_{\mathrm{i}}, \mathrm{c}_{\mathrm{i}}\right)$ are the generation cost, power generated, cost coefficients of the $\mathrm{i}^{\text {th }}$ generating unit of the plant respectively. Hence, the total production cost of the power plant for the 'n' generating unit

$$
\mathrm{F}_{\mathrm{T}}=\sum_{i=1}^{n} \cdot\left(\mathrm{F}_{\mathrm{i}}\left(\mathrm{P}_{\mathrm{i}}\right)=\sum_{i=1}^{n} \cdot\left(\mathrm{aiP}_{\mathrm{Gi}}{ }^{2}+\mathrm{biP}_{\mathrm{Gi}}+\mathrm{ci}\right)\right.
$$

\section{CONSTRAINTS APPLIED}

In power generating the main task to meet the load demand with minimum losses and at minimum cost. Basic meaning of an Economic load dispatch is that the generator's real and reactive power will be varied between the certain limits so as to meet a particular consumer demand with minimum fuel

Generally, there are two types of constraints: -

1.Equality Constraints: -These are the basic load flow equations.

2.Inequality Constraints: -

i) Generator Constraints: Each generating unit has a predefined minimum and maximum generation limit which should be satisfied while solving ELD.

$\mathrm{P}_{\min } \leq \mathrm{P} \leq \mathrm{P}_{\max }$

In total power the maximum reactive power can be determined by theheating of rotor and minimal reactive power is limited by the stability of the machine. So the generator reactive power $\mathrm{Q}$ should lie between these limits only to maintain system stability.

$$
\mathrm{Q}_{\min } \leq \mathrm{Q} \leq \mathrm{Q}_{\max }
$$

(ii) Voltage Constraints: At different nodes there are different values of voltage and phase angles. These values should be varied under certain limits. Due to transient stability concerns operating angle should lie in between $30 \mathrm{deg}$ and $45 \mathrm{deg}$. Stability increases due to the elevation of the operating angle.

(iii) Transmission Line Constraints: The thermal capability of the of the circuit depends upon the real as well as reactive power through the transmission line .

$$
\mathrm{Cp} \leq \mathrm{Cpmax}
$$

Where $\mathrm{C}_{\mathrm{pmax}}$ is the maximum loading capacity of the $\mathrm{P}^{\text {th }}$ line.

Apart from these, there are certain constraints on the tap setting of the power transformers used in the system and the running capacity limits. 


\section{COST FUNCTION}

Let $\mathrm{Ci}$ represent the cost, expressed in dollars per hour, of producing energy in the $\mathrm{i}^{\text {th }}$ generator.

$$
\mathrm{C}=\sum_{i=1}^{n} \cdot \mathrm{C}_{\mathrm{i}} \$ \mathrm{hr}(3)
$$

This equation describes the cumulative cost function of the power system for ' $n$ ' number of generators. $P_{i}$ has a significant effect on the cost. In order to increase the power generation rating of individual generators the torque of the prime mover concerned has to be increased but in doing so the amount of fuel used in increased considerably.

Reactive power $\mathrm{Q}_{\mathrm{i}}$ has negligible influence on the cost function. Considering these facts, the overall cost for all practical purposes is always taken to be a function of real power alone. This is shown in the equation below.

$$
\mathrm{C}=\sum_{i=1}^{n} \cdot \mathrm{Ci}(\mathrm{PGi})
$$

\section{STANDARD PARTICLE SWARM OPTIMIZATION}

A commonly used technique for Economic Load Dispatching is the Particle Swarm Optimization algorithm. Our per our research it is clearly evident that this fairly happens to be the best approach for solving the load dispatch problems and reducing the generation cost by a large margin. Under PSO there are again various approaches possible, all having the same base structure but with minor changes in the constraints or the value of coefficients or the way in which the solution space is optimized.

The PSO method was developed in the year 1995 by Dr.Eberhart and Dr.Kennedy. It was defined as a speculative optimization process which gets its inspiration from the characteristics of flocks of birds and a school of fish. The entire PSO operation is based on the movement of plausible solutions i.e. the particles in the solution space of the particular ELD problem. In contrast to the Genetic Algorithm, there exists no problem of crossover or mutation in PSO.

The PSO process is carried out over several hundreds of iterations. Each and every particle is evaluated against a fitness function and if the solution (particle) in question is found to be lower than all previous values associated with that particle then the current position is assigned to pbest (personal best).

The second "best" value determined is the global best or simply gbest. All the values of pbest are compared with each particle in the neighbourhood and the least amongst these is assigned to gbest.

After every iteration based on the pbest and gbest values the position and velocity of the particles are updated as per the vector configuration shown in Figure 2.

While updating the position and velocity the personal acceleration coefficient $\mathrm{cl}$ and the social acceleration coefficient c2 are also considered. Usually these have a value of 2 .

In the figure 2 the vector representation of the particles in accordance to their position and velocity is shown. Using this the updating of the position and velocity after each iteration is performed.

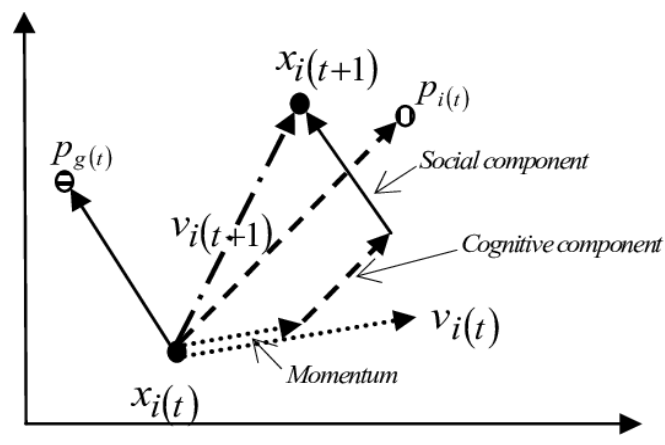

Fig.2: Vector Representation of Position and Velocity

In the exsisting work on PSO as in [1] the calculations have been performed on a Six-Generator System and the most economical value of Cost has been determined.

In the following table the initial data used for each of the six units is mentioned based on which the calculations are done.

\begin{tabular}{|c|c|c|c|c|c|c|c|c|c|c|}
\hline \multirow[b]{2}{*}{$\begin{array}{l}\text { uni } \\
\text { ts }\end{array}$} & \multicolumn{10}{|c|}{ Parameters } \\
\hline & $a_{i}$ & $\mathrm{~b}_{\mathrm{i}}$ & $\mathrm{c}_{\mathrm{i}}$ & $\mathrm{e}_{\mathrm{i}}$ & $\mathrm{f}_{\mathrm{i}}$ & $\mathrm{P}_{\text {ima }}$ & $\mathrm{P}_{\text {im }}$ & $P_{i}$ & $\mathrm{UR}_{\mathrm{i}}$ & $\bar{D}{ }_{i}$ \\
\hline 1 & .007 & 7 & $\begin{array}{c}24 \\
0\end{array}$ & $\begin{array}{c}30 \\
0\end{array}$ & .031 & 500 & $\begin{array}{c}10 \\
0\end{array}$ & $\begin{array}{c}44 \\
0\end{array}$ & 80 & 120 \\
\hline 2 & $\begin{array}{c}0.00 \\
9\end{array}$ & 10 & $\begin{array}{c}20 \\
0\end{array}$ & $\begin{array}{c}15 \\
0\end{array}$ & 0.063 & 200 & 50 & $\begin{array}{c}17 \\
0\end{array}$ & 50 & 90 \\
\hline 3 & $\begin{array}{c}0.00 \\
9\end{array}$ & 8.5 & $\begin{array}{c}22 \\
0\end{array}$ & $\begin{array}{c}20 \\
0\end{array}$ & 0.042 & 300 & 80 & $\begin{array}{c}20 \\
0\end{array}$ & 65 & 100 \\
\hline 4 & .009 & 11 & $\begin{array}{c}20 \\
0\end{array}$ & $\begin{array}{c}10 \\
0\end{array}$ & 0.08 & 150 & 50 & $\begin{array}{c}15 \\
0\end{array}$ & 50 & 90 \\
\hline 5 & $\begin{array}{c}.008 \\
0\end{array}$ & $\begin{array}{c}10 . \\
5\end{array}$ & $\begin{array}{c}22 \\
0\end{array}$ & $\begin{array}{c}15 \\
0\end{array}$ & 0.063 & 200 & 50 & $\begin{array}{c}19 \\
0\end{array}$ & 50 & 90 \\
\hline 6 & $\begin{array}{c}.007 \\
5\end{array}$ & 12 & $\begin{array}{c}19 \\
0\end{array}$ & $\begin{array}{c}10 \\
0\end{array}$ & 0.084 & 120 & 50 & $\begin{array}{c}11 \\
0\end{array}$ & 50 & 90 \\
\hline
\end{tabular}

Table 1: Data Used for PSO

Here $a_{i} b_{i}$ and $c_{i}$ are the cost coefficients respectively.

The results obtained after implementing various PSO algorithms on the given data is depicted below.

Table 2: Results of Existing Work on PSO

\begin{tabular}{c|c|c|c|}
\hline Power output(KW) & PSO & CPSO & MRSPSO \\
\hline P1 & 443.034 & 467.55 & 437.55 \\
\hline P2 & 169.03 & 163.05 & 165.44 \\
\hline P3 & 262.02 & 253.415 & 258.88 \\
\hline P4 & 134.78 & 115.07 & 134.43 \\
\hline P5 & 147.47 & 169.45 & 152.00 \\
\hline P6 & 125.37 & 113.24 & 98.91 \\
\hline Loss(KW) & 18.68 & 18.70 & 17.79 \\
\hline Total output(KW) & 1281.68 & 1281.77 & 1265.00 \\
\hline Total cost(\$/h) & 16372.9 & 16329.2 & $\mathbf{1 6 3 1 7 . 1 8}$ \\
\hline CPU Time(sec) & 0.6031 & 0.6105 & $\mathbf{0 . 3 2 1 5}$ \\
\hline
\end{tabular}

CPSO stands for Conventional Particle Swarm Optimization and MRSPSO stands for Moderate Random Search Particle Swarm Optimization.

\section{MODIFIED PARTICLE SWARM OPTIMIZATION}

In standard PSO algorithm, population size is chosen as a random value- usually 50, 100 or 150 . There is no specific constraint used while determining this value of population size. $w 1$ and w2 are the inertia weight constant and damping factor respectively, which have a value of 0.4 and 0.9 initially. 
Apart from this, there are two other coefficients namely, personal acceleration coefficient $\mathrm{c} 1$ and social acceleration coefficient $\mathrm{c} 2$ which each have a value of 2 which has been found to be the most accurate value.

To overcome the drawback of the random population size selection, a function has been determined wherein the inertia weight constant and damping factor w1 and w2 are employed to find a suitable value of the nPop (population size) for the 6-generator system being considered in this work.

The Flowchart depicting the process flow for Modified Particle Swarm Optimization has been shown in Figure 3 below.

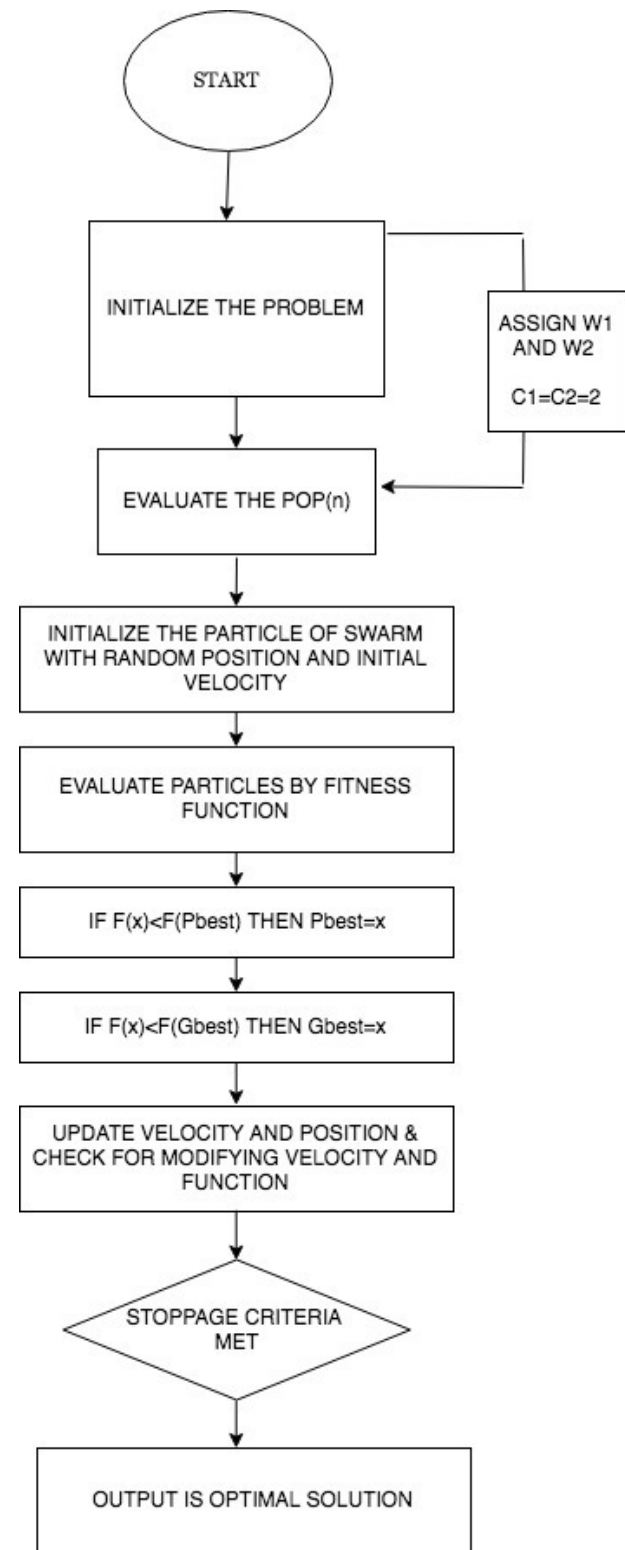

Fig.3: Flowchart for Modified Particle Swarm Optimization

The function developed is as follows: $\mathrm{nPop}($ new $)=\left[25 \mathrm{xe}^{2\left(\mathrm{w}_{1}+\mathrm{w}_{2}\right)}\right]$

For example, for $\mathrm{w}_{1}=0.4$ and $\mathrm{w}_{2}=0.9$ if $\mathrm{nPop}$ is randomly fixed at 100 the value for the total generation cost is coming up to be $8355 \$ / \mathrm{h}$.

Whereas, with $\mathrm{w}_{1}$ and $\mathrm{w}_{2}$ same as before but by determining nPop by the above equation we get nPop $=336$ and the total generation cost to be $8353.11 \$ / \mathrm{h}$.
The value of nPop should be less than 502 as per calculations based on the function above.

\section{PSO Method Parameters}

Population Size: Selected Based on The Above Equation Maximum No of Iterations: 1000

Inertia Weight Factor and Damping Factor $\mathrm{w}_{1}=0.9$ $\& \mathrm{w}_{2}=0.4$

Acceleration Constant: $\mathrm{C}_{1}=2 \& \mathrm{C}_{2}=2$

Limiting Criteria for $n P o p$

$\mathrm{N}(\text { pop })_{\text {new }}=\left[25 . \mathrm{e}^{2(\mathrm{w} 1+\mathrm{w} 2)}\right]$

Assuming $N(\text { pop })_{\text {new }}=\lambda$

$\lambda=25 . \mathrm{e}^{2(\mathrm{w} 1+\mathrm{w} 2)}$

Taking natural logarithms on both sides

nPop $($ new $)=\gamma$

$\gamma=25 . \mathrm{e}^{2\left(\mathrm{w}_{1}+\mathrm{w}_{2}\right)}$

$\ln (\gamma / 25)=2\left(\mathrm{w}_{1}+\mathrm{w}_{2}\right)$

$\mathrm{w}_{1}+\mathrm{w}_{2}<1.5$

Taking conditions for $\mathrm{w}_{1}+\mathrm{w}_{2}$;

$0.5 \ln (\gamma / 25)<1.5$

$\ln (\gamma / 25)<3$

$\gamma<\mathrm{e}^{3} .25$

Using GIF to get $\mathrm{Z}^{+}$values

$\lambda<502$

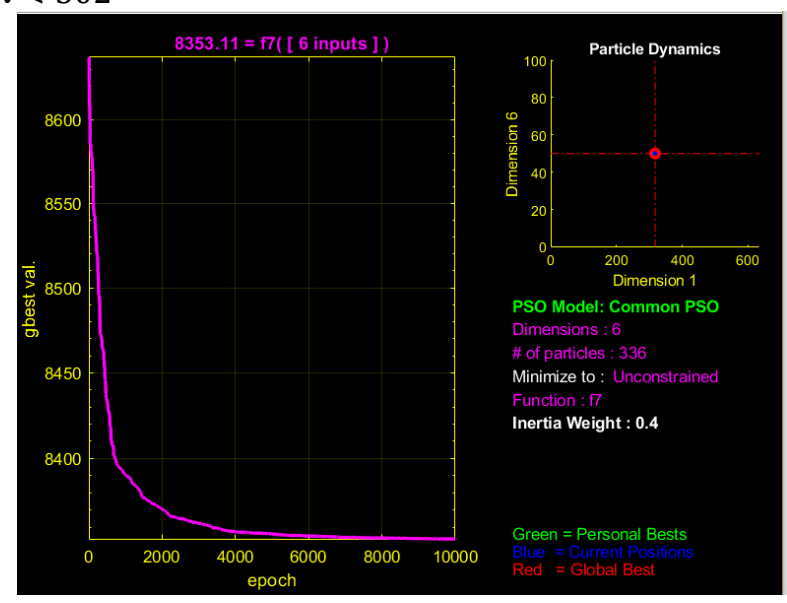

Fig. 4: Result for nPop=336 $($ w1 =0.9, w2=0.4)

\section{RESULTS}

As it is evident from the results shown below, there is a substantial change in the value of the total generation cost (\$/hour) when we use a carefully optimized value of the population size using the function described above, instead of randomly choosing the value as 100 .

Table 3: Results for Population Size 100 (Original)

\begin{tabular}{|l|l|l|l|l|l|}
\hline npop & a1 & a2 & w1 & w2 & cost \\
\hline 100 & 2 & 2 & 0.9 & 0.4 & 8356.37 \\
\hline 100 & 2 & 2 & 0.8 & 0.3 & 8359.27 \\
\hline 100 & 2 & 2 & 0.7 & 0.2 & 8358.80 \\
\hline 100 & 2 & 2 & 0.6 & 0.1 & 8352.81 \\
\hline
\end{tabular}

Published By 
Table 4: Results after using proposed function

\begin{tabular}{|l|l|l|l|l|l|}
\hline npop & a1 & a2 & w1 & w2 & cost \\
\hline 336 & 2 & 2 & 0.9 & 0.4 & 8353.11 \\
\hline 225 & 2 & 2 & 0.8 & 0.3 & 8353.27 \\
\hline 151 & 2 & 2 & 0.7 & 0.2 & 8354.99 \\
\hline 101 & 2 & 2 & 0.6 & 0.1 & 8357.2 \\
\hline
\end{tabular}

From the above comparison it seen evident that once the population size is calculated for each case specifically instead of choosing an arbitrary value of 100 the cost of generation of power decreases. For instance, at the initial conditions of $\mathrm{w} 1=0.9$ and $\mathrm{w} 2=0.4$, for $\mathrm{nPop}=100$ the cost is $8356.37 \$ / \mathrm{hr}$ while for $\mathrm{nPop}=336$ the value is $8353.11 \$ / \mathrm{hr}$. There is a decrease in cost of around $\$ 4$ which is quite significant on a large scale.

\section{CONCLUSION}

The technique that allocates active power outputs to generator units while complying with all the network constraints to ensure the most cost-effective set-up is referred to as economic load dispatch of a system. In this paper, modified particle optimization method has been analysed and its advantages over the existing methods in PSO have been shown. Instead of randomly choosing a value for the population size, an optimization function has been taken into account to find a custom population size, which in turn will reduce the cost of generation per hour. All the simulation has been performed using MATLAB tool. The results of modified PSO have been compared with those of existing works, it has been found that the obtained value is sufficiently lesser than previous existing values.

Advantages of modified PSO:

- Complex mathematical operations like gradient or matrix inversion such as those included in conventional techniques are not required. Only a fitness function that measures the 'quality' of the solution is needed. Hence, there is a removal of restrictions like differentiability, continuity, convexity, etc., that are usually imposed on the objective function. This in turn contributes to a decrease in complexity in the computational process.

- Since this is a population-oriented method, sensitivity to aideal initial solution is relatively low.

- Hybrid tools can be developed conveniently by incorporation of other already existing optimization tools.

- Since this method follows the rules of probabilistic transition, it has the tendency to escape local minima.

- Programming and modification can be easily applied to this technique using basic logical and mathematical operations.

- When measured in terms of memory and computational time, this method is lower in cost.

- Need for parameter tuning is comparatively low.

\section{Future Scope}

Modified Particle Swarm Optimization algorithm can be used to improve simple algorithms to improve the performance and give better results, which in turn would contribute towards the establishment of more cost-effective systems. This can be done by generating hybrid algorithms by incorporation of modified PSO into already existing algorithms used to solve ELD problems. The minimization function with voltage and reactive power constraints at various points on the system can be obtained by taking bus and line data as the input along with the load demand.

\section{REFERENCES}

1. Pabitra Mohan Dash, Asini Kumar and Sangram Keshori, "Economic Load Dispatch using Moderate Random Search PSO with Ramp Rate Limit Constraints" IEEE International Conference on Technologies for Smart-City Energy Security and Power (ICSESP-2018), March 28-30, 2018.

2. AbolfazlZaraki, and MohdFauzi Bin Othhman, "Implementing Particle Swarm Optimization Problem to solve Economic Load Dispatch Problem"- 2009 International Conference of Soft Computing and Pattern Recognition.

3. MaharnadNababAlarn, AkhileshMathur and KanhaiyaKurnar, "Economic Load Dispatch using a Differential Particle Swarm Optimization”- 1st IEEE International Conference on Power Electronics. Intelligent Control and Energy Systems (ICPEICES2016)

4. LoauTawfak Al-Bahrani, Jagdish C. Patra and Alex Stojcevski, "Solving economic load dispatch problem under valve point loading effects and generation constrains using a multi-gradient PSO algorithm"- 2018.

5. Pabitra Mohan Dash, Asini Kumar and SangramKeshori, "Solving Economic Load Dispatch Using Space Reduction Based PSO"-IEEE International Conference on Technologies for Smart-City Energy Security and Power (ICSESP-2018), March 28-30, 2018, Bhubaneswar, India.

6. M. Hamed, B. Mahdad ,K.Srairi and N.Mancer, "Solving Combined Economic Emission Dispatch Problem Using Time Varying Acceleration Based PSO"- 2012 IEEE.

7. YongqiangWang ,Jianzhong Zhou, Wen Xiao and Yongchuan Zhang, "Economic load dispatch of hydroelectric plant using a hybrid particle swarm optimization combined simulation annealing algorithm"2010 Second WRI Global Congress on Intelligent Systems.

8. KoustavDasgupta and Sumit Banerjee, "An Analysis of Economic Load Dispatch using DifferentAlgorithms"Proceedings of 2014 1st International Conference on Non Conventional Energy (ICONCE 2014)

9. Yong Wu and JingleiGuo, "Particle Swarm Optimization Using Levy Distribution for Economic Load Dispatch Problem"- 2017 International Conference on Industrial Informatics - Computing Technology, Intelligent Technology, Industrial Information Integration.

10. Mahamad NababAlam, "State-of-the-Art Economic Load Dispatch of Power Systems Using Particle Swarm Optimization"- Cornell University, 2018

11. VahidHosseinnezhad, Mehrdad Tarafdar Hagh and Ebrahim Babaei, "Quantum Particle Swarm Optimization for Economic Dispatch Problem with Valve-Point Effect", University of Tabriz Power energy system, vol. 29, no.10, pp. 720 - 730, 2007.

12. Manoj Mahajan and Shelly Vadhera, "Economic Load Dispatch of Different Bus Systems using Particle Swarm Optimization"- 2012. 
13. AnantBaijal, Vikram Singh Chauhan and T Jayabarathi, "Application of PSO, Artificial Bee Colony and Bacterial Foraging Optimization algorithms to economic load dispatch"- IJCSI International Journal of Computer Science Issues, Vol. 8, Issue 4, No 1, July 2011.

14. K.Vinodh, M.Jotmkumar, S.Sriramanivas and R.S. Dhivyaprakash, "An Efficient Particle Swarm.

15. S.Gautham and J. Rajamohan "Economic Load Dispatch using Novel Bat Algorithm”-2016 IEEE 1st International Conference on Power Electronics, Intelligent Control and Energy Systems (ICPEICES).

16. Yang, X.S.,"A New Metaheuristic Bat-Inspired Algorithm", Nature Inspired Cooperative Strategies for Optimization (NISCO2010): Studies in Computational Intelligence, Vol. 284, 2010, pp. 65-74.

17. Fister I et al., "Bat algorithm: Recent advances", IEEE 15th Int.Symposium on Computational Intelligence and Informatics, 2014, pp.163-167.

18. Wood Al, Wollenberg BF. Power Generation, Operation and Control. New York: Wiley; 1996, pp. 29-39 [chapter 3].

19. Nagarjuna Duvvuru "A Hybrid Interior Point Assisted Differential Evolution Algorithm for Economic Dispatch",

20. J.S. Al-Sumait, A.K. Al-Othman and J.K. Sykulski, "Application of pattern search method to power system valve point economic load dispatch", Elect. Power energy system, vol.29, no.10, pp.720-730, 2007.

21. Y.shi, R.Eberhart, "Parameter Selection in Particle Swarm Optimization,"-1998.

22. Application of Fireflies Algorithm to Solve Economic load Dispatch.

23. D.P. Kothari and I. J. Nagrath, "Modern Power System Analysis" or C. L. Wadhwa, "Electrical Power Systems"

24. Grainger J.J Stevenson and Jr W.D, "Power System Analysis"

25. Kothari D.P Nagrath I. J "Power System Engineering”

26. Power-System-Analysis-by-Hadi-Saadat-ElectricalEngineering. 